Provided for non-commercial research and education use. Not for reproduction, distribution or commercial use.

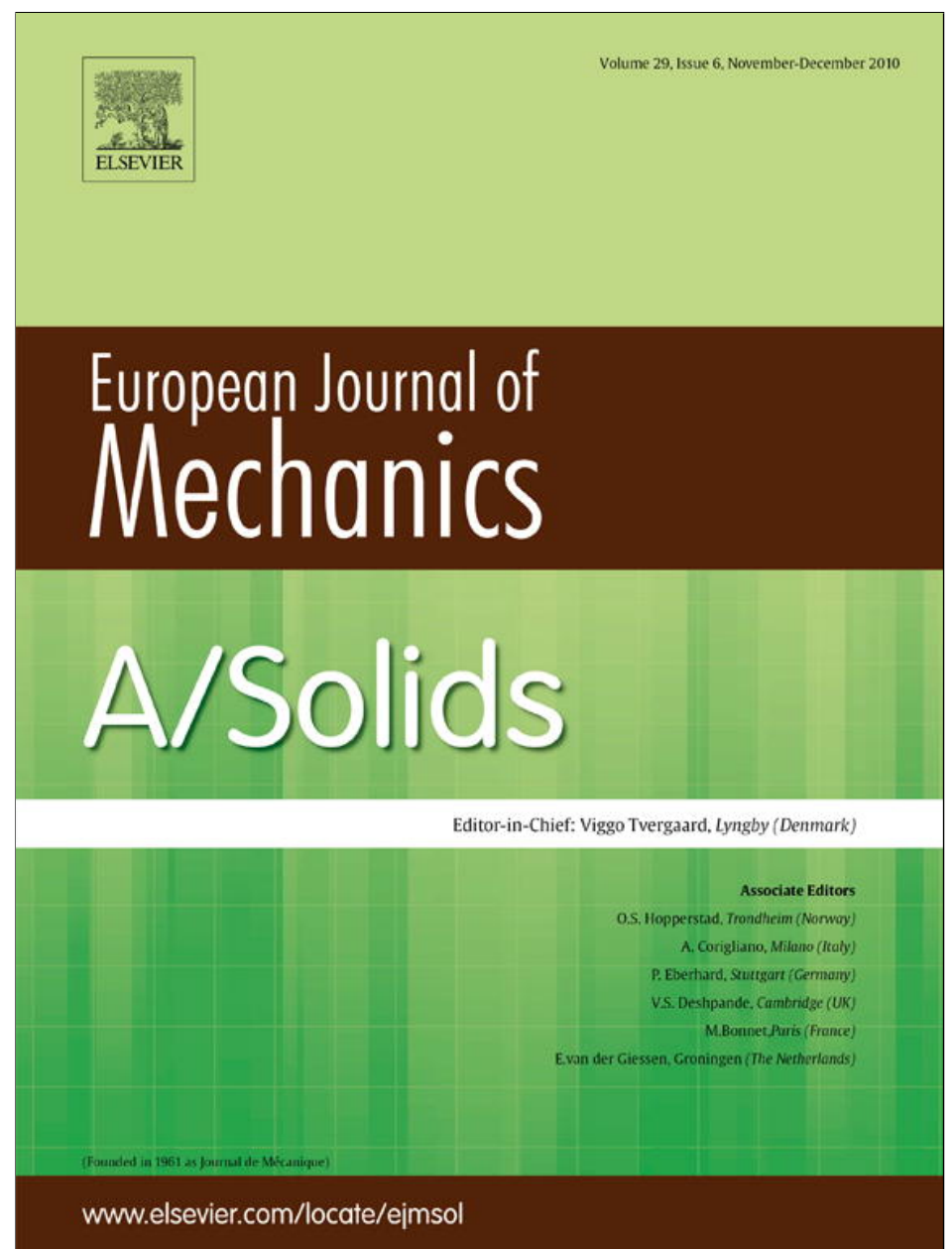

This article appeared in a journal published by Elsevier. The attached copy is furnished to the author for internal non-commercial research and education use, including for instruction at the authors institution and sharing with colleagues.

Other uses, including reproduction and distribution, or selling or licensing copies, or posting to personal, institutional or third party websites are prohibited.

In most cases authors are permitted to post their version of the article (e.g. in Word or Tex form) to their personal website or institutional repository. Authors requiring further information regarding Elsevier's archiving and manuscript policies are encouraged to visit:

http://www.elsevier.com/copyright 


\title{
Collisions and fractures: A predictive theory
}

\author{
Francesco Freddi ${ }^{\mathrm{a}, *}$, Michel Frémond ${ }^{\mathrm{b}}$ \\ a Laboratorio Lagrange, Dept. of Civil-Environmental Engineering, University of Parma, Parma, Italy \\ ${ }^{\mathrm{b}}$ Laboratorio Lagrange, Dept. of Civil Engineering, University of Tor Vergata, Roma, Italy
}

\section{A R T I C L E I N F O}

\section{Article history:}

Received 17 November 2009

Accepted 15 July 2010

Available online 24 July 2010

\section{Keywords:}

Collision

Principle of virtual work

Fracture

Damage

\begin{abstract}
A B S T R A C T
We investigate collisions of solids which can fracture. Equations of motion and constitutive laws provide a predictive theory. Assuming the collision as instantaneous, the equations of motion are derived from the principle of virtual work introducing new interior forces which describe the very large stresses and the very large contact forces resulting from the kinematic incompatibilities. They are interior volume percussion stresses and interior surface percussions both on the unknown fractures and on the colliding surface. In order to approximate these equations, we assume solids are damageable. In this point of view, it results that velocity is continuous with respect to space but its strain rate is very large in a thin region where the material is completely damaged, so approximating a fracture. When the velocity before collision is very large, the damaged zone may be large accounting for parts of the solid completely transformed into powder. The constitutive laws result from dissipative functions satisfying the second law of thermodynamics and able to model the fracturation phenomenon at the macroscopic engineering level. Representative numerical examples confirm that the model accounts for the fracturation qualitative properties.
\end{abstract}

(c) 2010 Elsevier Masson SAS. All rights reserved.

\section{Introduction}

Collisions and fractures of solids are important engineering issues, for instance, fragmentation of solids by blasting, by collisions, ... (Fourney, 1993; Romero, 2003). Besides, the demand for impact resistant design of structures crosses a wide spectrum.

The present work deals with collisions of deformable and damageable solids. In particular, we investigate the appearance of fractures due to impact phenomena. Collisions are phenomenona which occur in a very short period of time, involving large impulsive forces in the bodies. We limit the investigation of this problem at the engineering macroscopic level and derive a model by discontinuum mechanics theory (Frémond, 2007). We suppose that collision events are very short if compared with the duration of the other phenomena. Thus, we assume the collisions are instantaneous according to Frémond $(2002,2007)$. A collision is characterized by a time discontinuity of the velocity field: there is a smooth velocity before collision $\vec{U}^{-}$and the velocity $\vec{U}^{+}$after collision. This collision theory applies to collision of either rigid or deformable solids (Frémond, 2007), as well as of a solid colliding with an incompressible fluid (Frémond et al., 2003).

As a consequence of the collision fractures may appear in the solid. A fracture is characterized by a spatial discontinuity of the

\footnotetext{
* Corresponding author.

E-mail address: francesco.freddi@unipr.it (F. Freddi).
}

velocity after collision $\vec{U}^{+}$. We are not interested in the evolution of a fracture, but on its formation and this phenomenon is considered as instantaneous. Hence, we introduce different dissipative potentials as well as suitable unilateral conditions on fractures and along the boundary.

The predictive theory is based on the principle of virtual work giving the equations of motion and constitutive laws relating the internal forces to pertinent quantities describing the evolution. The constitutive laws introduce interior percussion stresses, ${ }^{1}$ and percussions which result from the kinematic incompatibilities. They are interior volume percussion stresses and interior surface percussions both on the unknown's fractures and on the colliding surfaces. The constitutive laws are derived with dissipative potentials which fulfill the second law of thermodynamics.

In order to solve numerically the resulting set of partial differential equations (reference is made to Bonetti and Frémond (2004b) and Frémond (2007) for details), we assume solids are damageable and that a damage quantity $\beta$ has value 1 when the material is undamaged and value 0 when it is completely damaged. We suppose that the damage quantity evolves rapidly in the collision: thus we assume it is discontinuous with respect to time at collision time: $\beta^{-}$before collision and $\beta^{+}$after collision. In this point of view, we may assume the velocity $\vec{U}^{+}$is continuous with respect to space but

\footnotetext{
1 Percussions are intended here as the variation of stress state or forces occurring during collision.
} 
its strain rate is very large in a layer where the damage quantity $\beta^{+}$is almost zero, i.e., a fracture is approximated by a thin damaged zone where $\beta^{+} \simeq 0$ (damage field is 1 where the material is not fractured). When the velocity before collision becomes very large, the damaged zone may be large accounting for parts of the solid completely transformed into powder where cohesion is totally lost.

The variational formulation of the equations of motion is discretized by the classical finite element technique. Moreover, kinematic constraints on the velocity field $\vec{U}$ are introduced in order to avoid overlapping phenomena along the contact surface and in the volume damaged zones. Finally, the discrete solution is obtained as a minimization of a nonconvex problem, reached through a specific numerical strategy that couples gradient descent technique (Ciarlet and Lions, 1990; Snyman, 2005) and the Uzawa's method (Zulehner, 2002). This scheme permits to account for the duality variables introduced by the constraints, for instance the impenetrability condition.

Several numerical simulations are proposed. Their aim is to demonstrate the capabilities of the predictive theory to describe qualitatively different failure modes occurring at collision time.

\section{The direct approach}

At collision time, the predictive theory is based on the principle of virtual work giving the equations of motion and constitutive laws relating the internal forces to pertinent quantities describing the evolution. The equations of motion introduce interior percussion stresses, and percussions which result from the kinematic incompatibilities. They are interior volume percussion stresses and interior surface percussions both on the unknown's fractures and on the colliding surfaces. ${ }^{2}$

For the sake of simplicity, let us consider a deformable solid $\Omega$ colliding a rigid fixed obstacle on $\partial \Omega_{1}$. Due to the kinematic incompatibilities very large interior forces appear within the solid, along the contact surface and on the unknown fracture surfaces. We consider the duration of the collision is very short, i.e., the time for the velocities to adapt to the presence of the obstacle is extremely short. Thus we assume the collision as instantaneous (time $t$ is fixed).

Very large interior forces become percussion stresses $\boldsymbol{\Sigma}$ and percussions $\vec{R}$. The percussions and percussion stresses can be thought as time concentrated quantities which define an atomic measure (a Dirac measure) intervening only at collision times. The different terms in the expression of the virtual work are linear functions of the virtual velocities. They involve the percussions and percussion stresses.

The system we consider at collision time is made of a solid $\Omega$ and an immobile obstacle $\partial \Omega_{1}$ which are in contact.

The system interior virtual work we choose is a linear function of the virtual strain rate and reads

$$
\begin{aligned}
\mathcal{T}_{\text {int }}(\vec{V})= & -\int_{\Omega / \Gamma} \sum: \mathbf{E}\left(\frac{\vec{V}^{+}+\vec{V}^{-}}{2}\right) \mathrm{d} \Omega \\
& -\int_{\partial \Omega_{1}} \vec{R} \cdot\left(\frac{\vec{V}^{+}+\vec{V}^{-}}{2}\right) \mathrm{d} \Gamma+\int_{\Gamma} \vec{R} \cdot\left\{\frac{\vec{V}^{+}+\vec{V}^{-}}{2}\right\} \mathrm{d} \Gamma,(1)
\end{aligned}
$$

where $\mathbf{E}(\vec{V}) \stackrel{\partial \Omega_{1}}{=} \nabla_{\text {sym }} \vec{V}$ is the classical symmetric strain rate tensor,

\footnotetext{
2 It is usual to derive the equation of motion through the principle of virtual power (Frémond, 2002). This principle can be used when all the quantities have densities with respect to the Lebesgue measure. When they have densities with respect to the atomic measure, as in the present situation, the principle of virtual power is advantageously replaced by the principle of virtual work. The principle of virtual work we use here, is not to be confused with the principle of virtual power where the velocities are understood as small displacements.
}

$\vec{V}^{+}$and $\vec{V}^{-}$are virtual velocity fields. The fracture $\Gamma$ is oriented: thus we consider a "left" part $\left(\vec{V}_{l}\right)$ and a "right" part $\left(\vec{V}_{r}\right)$ of the velocity field. So, the spatial velocity discontinuity i.e., the fracture velocity of deformation, is denoted by $\{\vec{V}\}=\vec{V}_{r}-\vec{V}_{l}$.

Because we assume the obstacle to be immobile, velocity $\vec{V}$ on contact surface $\partial \Omega_{1}$ is the gap velocity, i.e., the system deformation velocity on $\partial \Omega_{1}$.

The densities with respect to the Dirac measure are percussion stress tensors and interaction percussions between the solid and the obstacle as well as percussions between fracture surfaces. Percussions are generalized interior forces which appear when collisions occur. They are, as said earlier, usual interior forces concentrated in a very short period of time.

The virtual work of the acceleration forces is

$\mathcal{T}_{\text {acc }}(\vec{V})=\int_{\Omega} \rho[\vec{U}] \cdot\left(\frac{\vec{V}^{+}+\vec{V}^{-}}{2}\right) \mathrm{d} \Omega$,

where $\rho$ is the solid density and $[\vec{U}]=\vec{U}^{+}-\vec{U}^{-}$is the velocity discontinuity in the collision and $\rho[\vec{U}]$ is the collision inertial percussion. Let us note that the actual work of the acceleration forces is equal to the variation of the kinetic energy during collision.

\subsection{The equations of motion}

The principle of virtual work (Frémond, 2007)

$\forall \vec{V}, \mathcal{T}_{\text {acc }}(\vec{V})=\mathcal{T}_{\text {int }}(\vec{V})$,

gives the following equations of motion

$\rho\left(\vec{U}^{+}-\vec{U}^{-}\right)=\operatorname{div} \Sigma$, in $\Omega / \Gamma$,

$\{\Sigma\} \cdot \vec{N}=0, \Sigma \cdot \vec{N}=-\vec{R}$, on $\Gamma$,

$\Sigma \cdot \vec{N}=-\vec{R}$, on $\partial \Omega_{1}$

$\Sigma \cdot \vec{N}=0$, on $\partial \Omega / \partial \Omega_{1}$.

where vector $\vec{N}$ represents either the normal vector to fracture $\Gamma$ directed from the left toward the right or the outward normal vector to domain $\Omega$.

\subsection{The constitutive laws}

The constitutive laws are defined with dissipation functions: volume dissipation function $\Phi$, fracture dissipation function $\Phi_{s}$ and contact dissipation function $\Phi_{1}$. The dissipative functions are

$$
\begin{aligned}
\Phi\left(\mathbf{E}\left(\frac{\vec{U}^{+}+\vec{U}^{-}}{2}\right)\right)= & k_{0}\left\|\mathbf{E}\left(\frac{\vec{U}^{+}+\vec{U}^{-}}{2}\right)\right\|+k_{1}\left\|\mathbf{E}\left(\frac{\vec{U}^{+}+\vec{U}^{-}}{2}\right)\right\|^{2}, \\
\Phi_{S}\left(\left\{\frac{\vec{U}^{+}+\vec{U}^{-}}{2}\right\}\right)= & \sqrt{2} k_{2} \sqrt{\left|\left\{\frac{\vec{U}^{+}+\vec{U}^{-}}{2}\right\}\right|+k_{3}\left|\left\{\frac{\vec{U}^{+}+\vec{U}^{-}}{2}\right\}\right|} \\
& +I_{+}\left(\left\{\frac{\vec{U}^{+}+\vec{U}^{-}}{2}\right\} \cdot \vec{N}\right),
\end{aligned}
$$

where $\vec{N}$ is the normal vector to the fracture and

$\Phi_{1}\left(\frac{\vec{U}^{+}+\vec{U}^{-}}{2}, \frac{\vec{U}^{-}}{2}\right)=I_{-}\left(\vec{U}^{+} \cdot \vec{N}\right)$, 


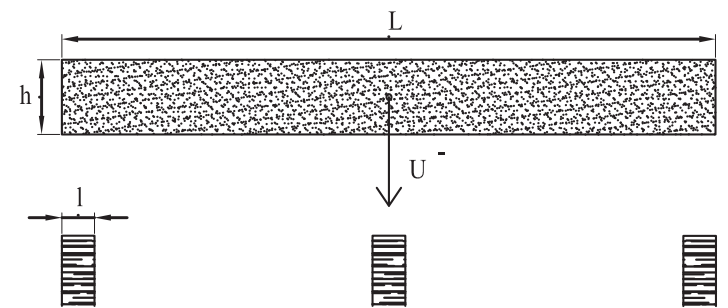

Fig. 1. Bar falling onto three rigid supports. The length of the bar is $L=4000 \mathrm{~mm}$ and ratio $L / h=20$ and $l / h=0.8$ have been assumed. The other parameters have the following value: $\mu=5 \cdot e 2 \mathrm{MPa}, \lambda=1 \cdot e 2 \mathrm{MPa}, \lambda s=1 \cdot e 2 \mathrm{MPa} / \mathrm{mm} \rho=3 \cdot e-5 \mathrm{~N} / \mathrm{mm}^{3}$ or $3 \cdot e-6 \mathrm{~N} / \mathrm{mm}^{3}, p=0.5, q=1.01, c=1 \cdot e 3 \mathrm{MPa} s, k=0.1 \mathrm{MPa} \mathrm{mm}{ }^{2}, w=2 \cdot e 3 \mathrm{MPa}$.

differently here $\vec{N}$ represents the outward normal vector to domain $\Omega$. For simplicity, we used these symbols to denote the length of a vector

$|\vec{x}|=\sqrt{x_{i} x_{i}}$,

and the norm of a symmetric tensor $\mathbf{A}$

$\|\mathbf{A}\|=\sqrt{A_{i j} A_{i j}}=\sqrt{\mathbf{A}^{2}}$.

The $k$ s are positive material constants. Volume dissipative function is a pseudo-potential of dissipation (Moreau, 1970; Halphen and Son, 1975). It insures a classical behaviour outside the fracture. Fracture dissipation function is not a pseudo-potential of dissipation. It implies that the behaviour is not progressive: a small collision does not produce a small fracture and a large collision does not produce a large fracture. On the contrary a small collision does not produce a fracture and a large collision produces a fracture. There is a threshold in the behaviour which results from function $\sqrt{2} k_{2} \sqrt{\left|\left\{\left(\vec{U}^{+}+\vec{U}^{-}\right) / 2\right\}\right|}$.

There is a competition between smooth strain rate measured by $k_{0}\left\|\mathbf{E}\left(\left(\vec{U}^{+}+\vec{U}^{-}\right) / 2\right)\right\|$ and nonsmooth strain rate measured by $\sqrt{2} k_{2} \sqrt{\left|\left\{\left(\vec{U}^{+}+\vec{U}^{-}\right) / 2\right\}\right|}$. Thus if $k_{2}$ is large compared to $k_{0}$ it is difficult to have fractures and if it is small, fractures appear easily. Thus parameter $k_{2}$ is a threshold for fractures to appear. Moreover, the latter function avoids the appearance of too many fractures characterized by small velocity discontinuities (square root function, with property $\sqrt{a+b} \leq \sqrt{a}+\sqrt{b}$, forbids a fracture with amplitude $a+b$ to be replaced by two small fractures with amplitudes $a$ and $b$ ).

The functions $I_{-}, I_{+}$are the indicator functions of $\left.]-\infty, 0\right]=\mathbb{R}^{-}$, $\left(I_{-}(\gamma)=0\right.$, if $\gamma \leq 0$ and $I_{-}(\gamma)=+\infty$, if $\left.\gamma>0\right)$, and of $\left[0, \infty\left[=\mathbb{R}^{+}\right.\right.$, $\left(I_{+}(\gamma)=0\right.$, if $\gamma \geq 0$ and $I_{+}(\gamma)=+\infty$, if $\gamma<0$ ), (see Moreau, 2003) respectively. Indicator function $I_{+}$takes into account the noninterpenetration condition along the fracture

$\left\{\vec{U}^{+}\right\} \cdot \vec{N} \geq 0$

Function $\Phi_{1}$ introduces the non-interpenetration condition on contact surface $\partial \Omega_{1}$. Function $\Phi_{s}$ is split in a convex part, $\Phi_{s}^{c}$ and a differentiable nonconvex part, $\Phi_{s}^{n c}$. Its generalized derivative is the sum of the sub-differential of the convex part and of the extended derivative of the nonconvex part

$$
\begin{aligned}
\partial \Phi_{s}\left(\left\{\frac{\vec{U}^{+}+\vec{U}^{-}}{2}\right\}\right)= & \frac{\partial \Phi_{s}^{n c}}{\partial\left\{\left(\vec{U}^{+}+\vec{U}^{-}\right) / 2\right\}}\left(\left\{\frac{\vec{U}^{+}+\vec{U}^{-}}{2}\right\}\right) \\
& +\partial \Phi_{s}^{c}\left(\left\{\frac{\vec{U}^{+}+\vec{U}^{-}}{2}\right\}\right) .
\end{aligned}
$$

The constitutive laws are

$$
\begin{aligned}
& \Sigma \in \partial \Phi\left(\mathbf{E}\left(\frac{\vec{U}^{+}+\vec{U}^{-}}{2}\right)\right), \\
& -\vec{R} \in \partial \Phi_{S}\left(\left\{\frac{\vec{U}^{+}+\vec{U}^{-}}{2}\right\}\right), \text { on } \Gamma, \vec{R} \in \partial I_{-}\left(\vec{U}^{+} \cdot \vec{N}\right), \text { on } \partial \Omega_{1},
\end{aligned}
$$

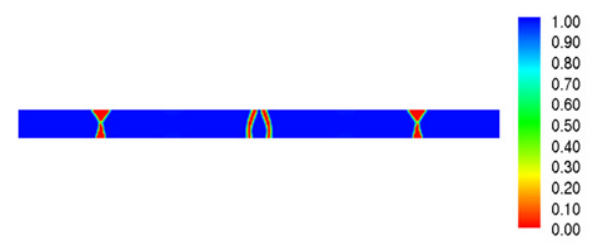

$1.5625 \mathrm{~m} / \mathrm{s}$

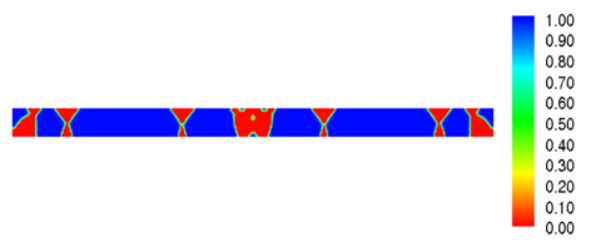

$6.25 \mathrm{~m} / \mathrm{s}$

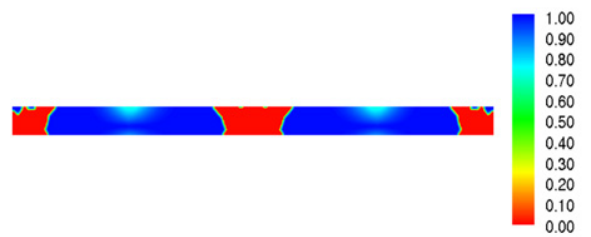

$25 \mathrm{~m} / \mathrm{s}$

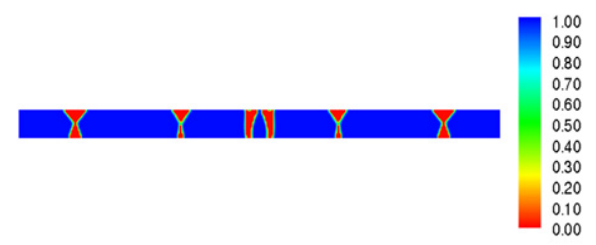

$3.125 \mathrm{~m} / \mathrm{s}$

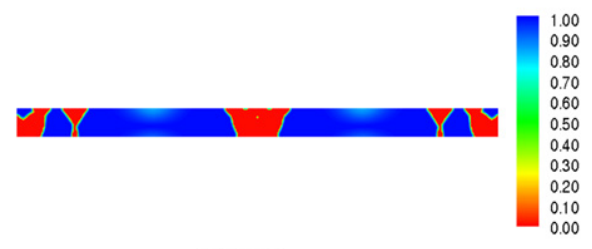

$12.5 \mathrm{~m} / \mathrm{s}$

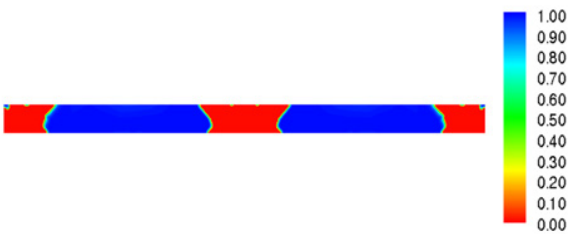

$50 \mathrm{~m} / \mathrm{s}$

Fig. 2. Damage in a heavy bar falling at different velocities. The blue zone is not damaged. The thin red zones are damaged. They account for fractures. When the falling velocity is very large, the damaged zones become completely fragmented domains. (For interpretation of the references to colour in this figure legend, the reader is referred to the web version of this article). 


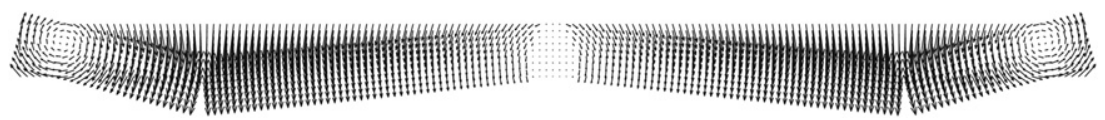

Fig. 3. Heavy bar: velocity field $\vec{U}^{+}$after the collision for $U^{-}=1.5625 \mathrm{~m} / \mathrm{s}$. The bar is broken into five pieces which do not bounce on the obstacles.

with

$$
\begin{aligned}
\partial \Phi_{s}(\vec{X}) & =\frac{\partial \Phi_{s}^{n c}}{\partial \vec{X}}(\vec{X})+\partial \Phi_{s}^{c}(\vec{X}) \\
& =\left\{\begin{aligned}
\left(\frac{k_{2}}{\sqrt{2} \sqrt{|\vec{X}|}}+k_{3}\right) \frac{\vec{X}}{|\vec{X}|}, & \text { if }|\vec{X}|>0, \\
\mathbb{R}^{3}, & \text { if } \vec{X}=0 .
\end{aligned}\right.
\end{aligned}
$$

Let us note that when discontinuity $\left\{\left(\vec{U}^{+}+\vec{U}^{-}\right) / 2\right\}$ is null, percussion $\vec{R}$ may assume any value, this value being given by relationship (3), $\vec{R}=-\Sigma \cdot \vec{N}$. In this situation, there is no fracture and the volume constitutive law (7) is valid and conveys the mechanical information. Fracture constitutive law (8) is also valid but conveys no mechanical information.

The mechanical aspects of collisions have an important influence on thermal behavior but the thermal aspects have a limited influence on the mechanical behavior. Thus it is reasonable to assume the temperature does not intervene in the mechanical equations. Following Frémond (2007), the thermal equations involving the dissipated work may be solved to get temperature $T^{+}$ after collision.

\subsection{The direct problem}

It may be proved that there are velocities after collision which minimize a functional

$$
\inf \left\{\mathcal{F}(\vec{V}) \mid \vec{V} \in \operatorname{SBD}(\Omega), \vec{V}=0 \text { outside } \partial \Omega_{1}\right\},
$$

over the set $\operatorname{SBD}(\Omega)$ of the special bonded deformation velocities (those functions are smooth outside the fractures and discontinuous on the fractures) (Ambrosio et al., 2000; Ambrosio et al., 1997; Attouch et al., 2004; Braides, 1998). The functional $\mathcal{F}(\vec{V})$ is

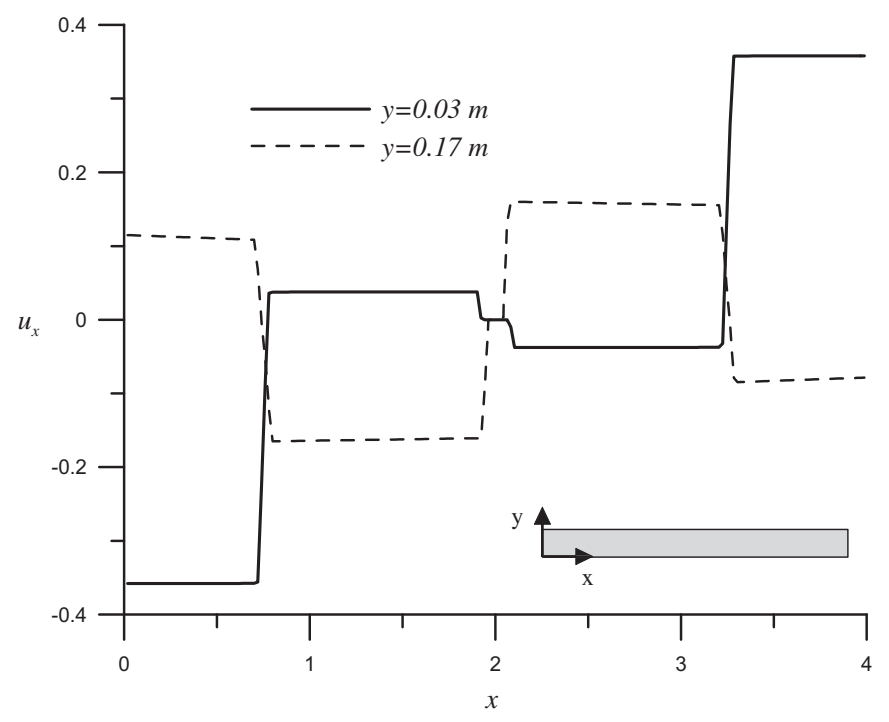

Fig. 4. Heavy bar: horizontal velocity $U_{x}^{+}$along two straight horizontal lines located at different positions for $U^{-}=1.5625 \mathrm{~m} / \mathrm{s}$. The vertical distances $y$ are measured from the bottom of the beam with thickness $h=0.2 \mathrm{~m}$. The strong discontinuities in the velocity values outlined the fractures while the remaining pieces of the solids undergo a nearly rigid body motions. Note the null velocity of the central piece on the central obstacle.

$$
\begin{aligned}
\mathcal{F}(\vec{V})= & \int_{\Omega / \Gamma} \Xi\left(\mathbf{E}\left(\vec{V}+\vec{U}^{-}\right)\right) \mathrm{d} \Omega+\int_{\Gamma} \Xi_{S}\left(\left\{\vec{V}+\vec{U}^{-}\right\}\right) \mathrm{d} \Gamma \\
& +\int_{\partial \Omega_{1}} \Xi_{1}(\vec{V}) \mathrm{d} \Gamma+\int_{\Omega} \frac{\rho}{2}(\vec{V})^{2}-\rho \vec{V} \cdot \vec{U}^{-} \mathrm{d} \Omega,
\end{aligned}
$$

where functions $\Xi, \Xi_{s}$ and $\Xi_{1}$ are defined according functions $\Phi, \Phi_{s}$ and $\Phi_{1}$

$$
\begin{aligned}
& \Xi\left(\mathbf{E}\left(\vec{V}+\vec{U}^{-}\right)\right)=k_{0}\left\|\mathbf{E}\left(\vec{V}+\vec{U}^{-}\right)\right\|+\frac{k_{1}}{2}\left\|\mathbf{E}\left(\vec{V}+\vec{U}^{-}\right)\right\|^{2}, \\
& \Xi_{s}\left(\left\{\vec{V}+\vec{U}^{-}\right\}\right)=2 k_{2} \sqrt{\left|\left\{\vec{V}+\vec{U}^{-}\right\}\right|}+k_{3}\left|\left\{\vec{V}+\vec{U}^{-}\right\}\right| \\
& \Xi_{1}(\vec{V})=I_{-}(\vec{V} \cdot \vec{N}) \text {. } \\
& +I_{+}\left(\left\{\vec{V}+\vec{U}^{-}\right\} \cdot \vec{N}\right) \text {, }
\end{aligned}
$$

This problem has been analyzed in Bonetti and Frémond (2004b). It was proved that problem (9) has solutions which give both the fractures (which are a priori unknown) and the velocities. Besides, in Bonetti and Frémond (2004a) a $1-D$ example is analyzed and closed form solutions are described.

\section{The regularized approach}

Numerical solution of the previous problem presents a major difficulty due to the unknown positions of fracture surfaces $\Gamma$ which are free discontinuities. So, we propose a regularized approach in the context of fracture damage mechanics. In particular, we introduce a spatial damage variable $\beta \in[0,1]$ that approximately describes the zone where the material is fractured. In fact, $\beta=1$ represents the sound material while $\beta=0$ is equivalent to the completed damaged state. The main idea, firstly proposed in Frémond (1987) and Frémond and Nedjar (1996), is based on adaptation of the principle of virtual work. In particular, we assume that damage results from microscopic motions, and includes the work of these motions in the principle of virtual work. This contribution is assumed to depend on the rate of damage and on the rate of the damage gradient. This last term is introduced to account for the local interaction of the damage at a material point on the damage of its neighborhood. So, the internal virtual work (1) is replace by

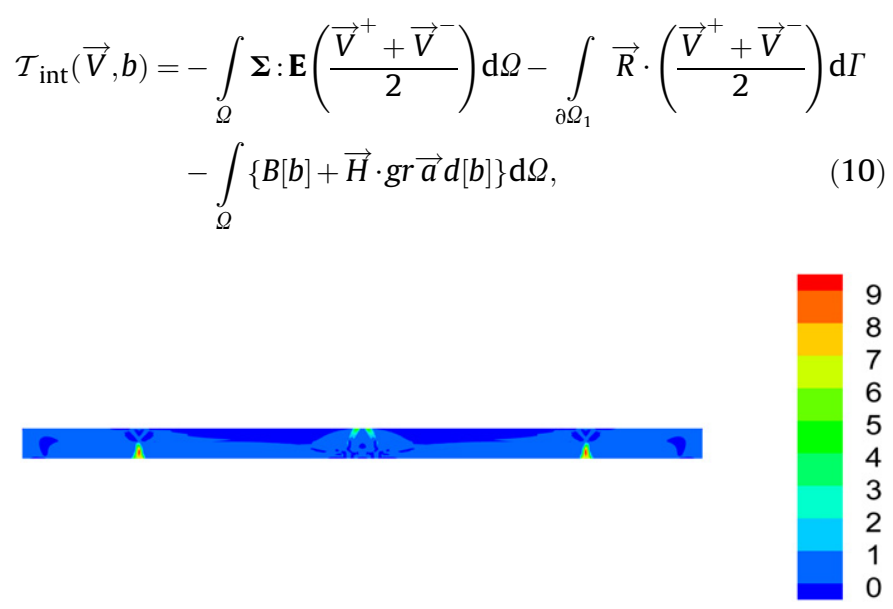

Fig. 5. Heavy bar: divergence of the velocity vector after the collision for $U^{-}=1.5625 \mathrm{~m} / \mathrm{s}$. The opening fractures are represented by zone with high values of $\operatorname{div} \vec{U}^{+}$. 

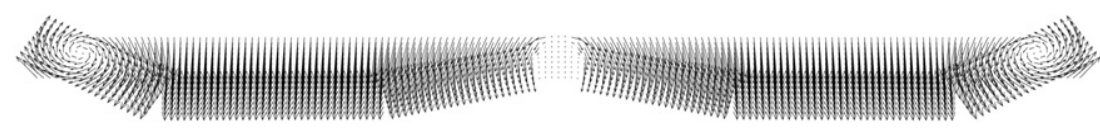

Fig. 6. Heavy bar: velocity $\vec{U}^{+}$after the collision for $U^{-}=3.125 \mathrm{~m} / \mathrm{s}$. The bar is broken into seven pieces.

being $[b]=b^{+}-b^{-}$, where $\vec{V}^{+}, \vec{V}^{-}$and $b^{+}, b^{-}$are virtual velocities. The first and the second terms have already been introduced and are related to the mechanical action and reactions. The third and fourth terms are unusual: $B$ is a mechanical work, in particular the internal damage work which is responsible for the evolution of the damage during the collision and $\vec{H}$ is a flux vector responsible for the interaction of the damage at a point on the damage on its neighborhood.

\subsection{The equations of motion}

The principle of virtual work

$\forall \vec{V}, b \quad \mathcal{T}_{\text {acc }}(\vec{V})=\mathcal{T}_{\text {int }}(\vec{V}, b)$,

gives two sets of equations of motion. By choosing convenient virtual velocity, we get

$\rho[\vec{U}]=\operatorname{div} \Sigma$, in $\Omega$,

$\Sigma \cdot \vec{N}=-\vec{R}$, on $\partial \Omega_{1}$

$\Sigma \cdot \vec{N}=0$, on $\partial \Omega / \partial \Omega_{1}$,

and

$\operatorname{div} \vec{H}-B=0$, in $\Omega$,

$\vec{H} \cdot \vec{N}=0$, on $\partial \Omega$,

where $\vec{N}$ is the outward normal to $\Omega$. The equations (12) and (13) account for macroscopic and microscopic effects.

\subsection{The constitutive laws}

In our problem we assume that the velocity $\vec{U}^{-}$, supposed for the sake of simplicity to be a rigid body velocity, and the damage $\beta^{-}$before the collision are assigned and let the unknowns be the velocity $\vec{U}^{+}$and the damage $\beta^{+}$after the collision. The constitutive laws, which have to satisfy the second law of thermodynamics, for the mechanical and damage interior forces are defined by three functions: the volume free energy $\Psi$, the volume dissipative function $\Phi$ and along the contact surface with the obstacle pseudopotential of dissipation $\Phi_{s}$. We have to choose them in such a way that $\beta^{+}$is zero in thin layers and 1 elsewhere and that the strain rate is very large in the thin layer and almost zero elsewhere. We choose the volume free energy as

$\Psi(\varepsilon, \beta, \operatorname{grad} \beta)=w(1-\beta)+\frac{k}{2}(\operatorname{grad} \beta)^{2}+\frac{\beta}{2} \varepsilon \mathbb{C} \varepsilon+I(\beta)$.

The volume dissipative function is addressed as follows

$$
\begin{aligned}
& \Phi\left(\mathbf{E}\left(\frac{\vec{U}^{+}+\vec{U}^{-}}{2}\right),[\beta], \operatorname{grad}[\beta]\right)=\frac{c}{2}[\beta]^{2}+\frac{\mu}{q}\left(\beta^{-}+\frac{[\beta]}{2}\right) \\
& \left\|\mathbf{E}\left(\frac{\vec{U}^{+}+\vec{U}^{-}}{2}\right)\right\|^{q}+\frac{\lambda}{r}\left\|\mathbf{E}\left(\frac{\vec{U}^{+}+\vec{U}^{-}}{2}\right)\right\|^{r}+I_{-}([\beta]) \\
& \quad+I_{+}\left(\operatorname{div}\left(\frac{\vec{U}^{+}+\vec{U}^{-}}{2}\right)\right) .
\end{aligned}
$$

The contact surface with the obstacle pseudo-potential of dissipation is
$\Phi_{s}\left(\frac{\vec{U}^{+}+\vec{U}^{-}}{2}\right)=\lambda_{s}\left(\frac{\vec{U}^{+}+\vec{U}^{-}}{2}\right)^{2}+I_{-}\left(U_{N}^{+}\right)$,

where $U_{N}=\vec{U} \cdot \vec{N}$ is the normal velocity.

Quantity $w$ is the cohesion of the material, $\mathbb{C}$ is the classical elasticity tensor, $\varepsilon$ the deformation tensor which does not change during collision and $k$ is the damage coefficient which quantifies the influence of the damage at a point onto the damage of its neighborhood. The extension parameter $k$ controls the size of the transition zone between sound material and damaged material. If $k$ is large, damage is diffuse and spread in the whole domain. Contrarily, if $k$ is small, the damage is concentrated in thin zones which may represent fractures. The values of $k$ can be measured with structure experiments, but not with sample experiments where the state quantities are homogeneous.

The parameters $c, \lambda, \mu, \lambda_{s}$ characterize the microscopic and macroscopic dissipations. The exponents $r \in] 0,1[$ and $q \in[1,2]$ indicate the nature of the behaviour of the constitutive law: convex or concave. In particular, the convex term involving the strain rate $\mathbf{E}$ consists of the visco-plastic Norton-Hoff-Friaa (Friaa, 1979), potential which approximates the perfect plastic potential as $q$ tends to one. Moreover, the effect of the concave term with power $r$ lower than 1 is to avoid having many regions with small discontinuities and to have the strain rates very large in thin damaged zones.

The function $I$ is the indicator function of the intervals $[0,1]$, $(I(\gamma)=0$, if $0 \leq \gamma \leq 1$ and $I(\gamma)=+\infty$, if $\gamma \notin[0,1])$. The indicator functions $I, I_{-}, I_{+}$take care of the internal constraints on the damage variable and on the velocity field.

$\beta \in[0,1] ; \quad[\beta] \leq 0 ; \quad U_{N}^{+} \leq 0$, on $\partial \Omega_{1} ; \operatorname{div}\left(\frac{\vec{U}^{+}+\vec{U}^{-}}{2}\right) \geq 0$ in $\Omega$.

The first internal constraint results from the definition of damage. The second constraint means that the solid cannot mend during the collision. The other constraints are related to the velocity field and take into account impenetrability conditions: the first one is on the obstacle contact surface and the last one, following an idea of Jean Jacques Moreau (1966b), replaces the impenetrability condition on the fractures by a volume nonoverlapping condition. This relationship results from mass balance relationships $\operatorname{div} \vec{U}^{-}=0$ and $\operatorname{div} \vec{U}^{+}=0$ in the nonfractured domain (assuming the density to be constant) and from impenetrability condition (6), $\left\{\vec{U}^{+}\right\} \cdot \vec{N} \geq 0$.

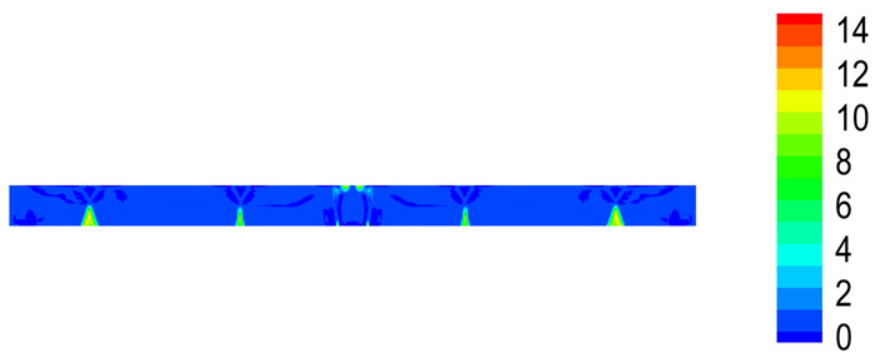

Fig. 7. Heavy bar: divergence of the velocity vector after the collision for $U^{-}=3.125$. The opening fractures are represented by zone with high values of $\operatorname{div} \vec{U}^{+}$. 


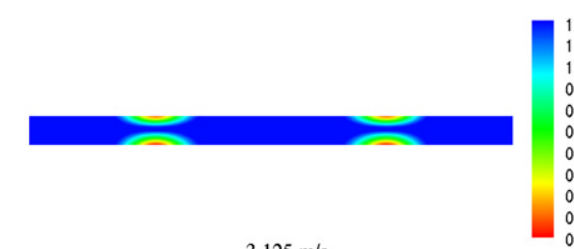

$3.125 \mathrm{~m} / \mathrm{s}$

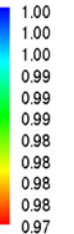

1
0.9
0.8
0.7
0.6
0.5
0.4
0.3
0.2
0.1
0

$12.5 \mathrm{~m} / \mathrm{s}$

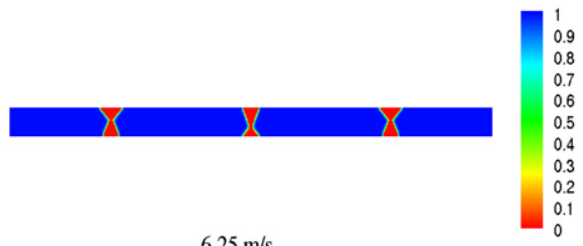

$6.25 \mathrm{~m} / \mathrm{s}$

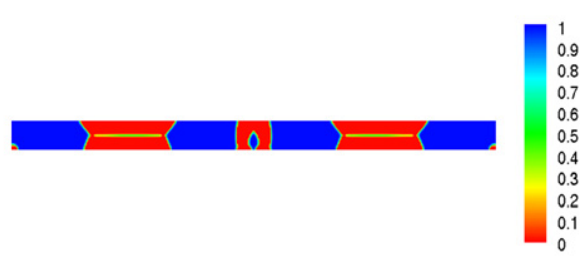

$25 \mathrm{~m} / \mathrm{s}$

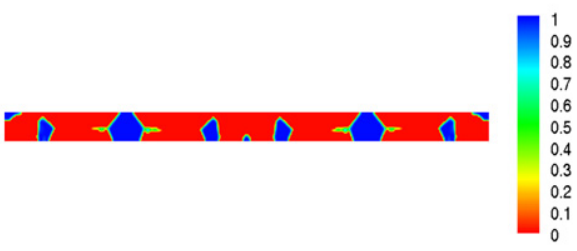

$100 \mathrm{~m} / \mathrm{s}$

Fig. 8. Damage in a light bar falling at different velocities (The damage scale depends on the velocity). The blue zone is not damaged. The thin red zones are damaged. They account for fractures. When the falling velocity is very large, the damaged zones become completely fragmented domains. (For interpretation of the references to colour in this figure legend, the reader is referred to the web version of this article).

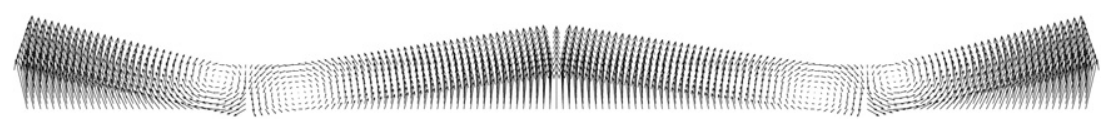

Fig. 9. Light bar: velocity $\vec{U}^{+}$after the collision for $U^{-}=6.25 \mathrm{~m} / \mathrm{s}$. The bar is broken into four pieces which bounce on the obstacles.

The previous functions give the following set of constitutive laws

$$
\begin{gathered}
\sum=\lambda\left\|\mathbf{E}\left(\frac{\vec{U}^{+}+\vec{U}^{-}}{2}\right)\right\|^{r-2} \mathbf{E}\left(\frac{\vec{U}^{+}+\vec{U}^{-}}{2}\right)+\mu\left(\frac{\beta^{-}+\beta^{+}}{2}\right) \\
\left\|\mathbf{E}\left(\frac{\vec{U}^{+}+\vec{U}^{-}}{2}\right)\right\|^{q-2} \mathbf{E}\left(\frac{\vec{U}^{+}+\vec{U}^{-}}{2}\right)-p \mathbf{I},
\end{gathered}
$$

$$
\begin{aligned}
B \in & -w+\frac{1}{2} \varepsilon \mathbb{C} \varepsilon+\partial I\left(\beta^{+}\right)+c[\beta]+\frac{\mu}{2 q}\left\|\mathbf{E}\left(\frac{\vec{U}^{+}+\vec{U}^{-}}{2}\right)\right\|^{q} \\
& +\partial I_{-}([\beta]),
\end{aligned}
$$

$\vec{H}=k \operatorname{gr} \vec{a} d \beta^{+}$,

$\vec{R} \in \lambda_{s}\left(\vec{U}^{+}+\vec{U}^{-}\right)+\partial I_{-}\left(U_{N}^{+}\right) \vec{N}$,

where

$-p \in \partial I_{+}\left(\operatorname{div}\left(\frac{\vec{U}^{+}+\vec{U}^{-}}{2}\right)\right)$.

The sub-differential sets $\partial I_{+}$and $\partial I_{-}$of the indicator functions of the positive and negative numbers $\mathbb{R}^{+}$and $\mathbb{R}^{-}$are defined as: $\partial I_{+}(0)=\mathbb{R}^{-}, \partial I_{+}(\gamma)=0$ for $\gamma>0$ and $\partial I_{+}(\gamma)=\varnothing$ for $\gamma<0, \partial I_{-}(0)=\mathbb{R}^{+}$, $\partial I_{-}(\gamma)=0$ for $\gamma<0$ and $\partial I_{-}(\gamma)=\varnothing$ for $\gamma>0$. The quantity $\partial I_{-}\left(U_{N}^{+}\right) \vec{N}$ is the impenetrability percussion reaction. It is active only if the other interactions are not sufficient for the solid and the obstacle not to interpenetrate, i.e., when the normal velocity after the collision is 0 . The quantity $-p$ is the internal impenetrability pressure. This reaction prevents the volume overlapping. From the numerical simulations proposed in Section 4 clearly outcomes that $-p$ is active only in the fractured zones in compression or in the fragmented ${ }^{3}$ regions, avoiding interpenetration. In fact, the undamaged portions of the solid after collision have nearly rigid body velocity, in agreement with the rigid-plastic component of the constitutive law.

Let us note that the second law of thermodynamics equivalent to the following inequality is satisfied for all admissible values of $r$ and $q$ (Frémond, 2007)

$$
\begin{aligned}
& \sum: \mathbf{E}\left(\frac{\vec{U}^{+}+\vec{U}^{-}}{2}\right)+B[\beta]+\vec{H} \cdot \operatorname{gr} \vec{a} d[\beta]-[\Psi] \geq \mu\left(\frac{\beta^{-}+\beta^{+}}{2}\right) \\
&\left\|\mathbf{E}\left(\frac{\vec{U}^{+}+\vec{U}^{-}}{2}\right)\right\|^{q}+\lambda\left\|\mathbf{E}\left(\frac{\vec{U}^{+}+\vec{U}^{-}}{2}\right)\right\|^{r}-w[\beta]+c[\beta]^{2} \\
&+\frac{\mu}{2 q}[\beta]\left\|\mathbf{E}\left(\frac{\vec{U}^{+}+\vec{U}^{-}}{2}\right)\right\|^{q}+\partial I_{-}([\beta])[\beta]+\partial I\left(\beta^{+}\right)[\beta] \\
&+k \operatorname{gr} \vec{a} d \beta^{+} \cdot \vec{g} \operatorname{rad}[\beta] \geq 0 .
\end{aligned}
$$

\footnotetext{
${ }^{3}$ The difference between fractured and fragmented states consists in the fact that in the first case cracks are thin and well separated while in the second case the rupture is completely diffused and well defined cracks cannot be appreciated.
} 


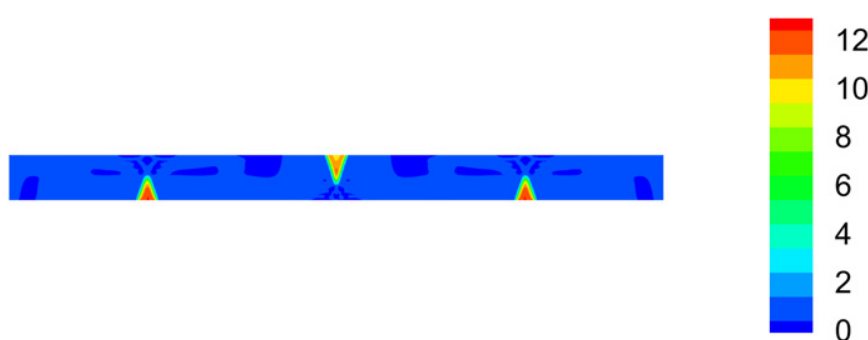

Fig. 10. Light bar: divergence of the velocity vector after the collision for $U^{-}=6.25 \mathrm{~m} /$ s. The opening fractures are represented by zone with high values of $\operatorname{div} \vec{U}^{+}$.

\subsection{A simplified problem: the equation}

The principle of virtual work and a proper use of the constitutive laws lead to two sets of equations and permit to compute the velocity and damage of the body after the collision depending on the incoming velocity and the damage state before the collision.

For simplicity, we assume the body undamaged before the collision, that its velocity is a rigid body velocity and that it is not deformed before collision.

$\beta^{-}=1, \quad \mathbf{E}\left(\vec{U}^{-}\right)=0, \quad \varepsilon=0$.

Moreover, assuming (24) it results

$\partial I\left(\beta^{+}\right)+\partial I-\left(\beta^{+}-1\right)=\partial I\left(\beta^{+}\right)$.

So, the equations needed to find $\vec{U}^{+}$and $\beta^{+}$are

$\rho \vec{U}^{+}-\operatorname{div} \sum\left(\vec{U}^{+}\right)=\rho \vec{U}^{-}$, in $\Omega$,

$\sum \cdot \vec{N}+\lambda_{s}\left(\vec{U}^{+}+\vec{U}^{-}\right)+\partial I_{+}\left(U_{N}^{+}\right) \vec{N} \ni 0$, on $\partial \Omega_{1}$

$\sum \cdot \vec{N}=0$, on $\partial \Omega / \partial \Omega_{1}$,

$c \beta^{+}-k \Delta \beta^{+}+\partial I\left(\beta^{+}\right) \ni w+c-\frac{\mu}{2 q}\left\|\mathbf{E}\left(\frac{\vec{U}^{+}}{2}\right)\right\|^{q}$, in $\Omega$,

$\frac{\partial \beta^{+}}{\partial N}=0$, on $\partial \Omega$

\section{Numerical examples}

In this section three numerical experiments are presented. Velocity $\vec{U}^{-}$is given and velocity $\vec{U}^{+}$is computed. Note that there is no time step because collision is instantaneous. Let us emphasize that we do not try to compare our results with those of actual experiments (even if the last example shows that the theory describes at the qualitative level an actual experiment) but we want to illustrate the capability of the proposed model to describe the different failure modes.

After collision the material may present three different states: not fractured, fractured and fragmented. In details, the not fractured region is represented by the undamaged zone $(\beta=1)$, the fractured state presents completed damaged materials with an

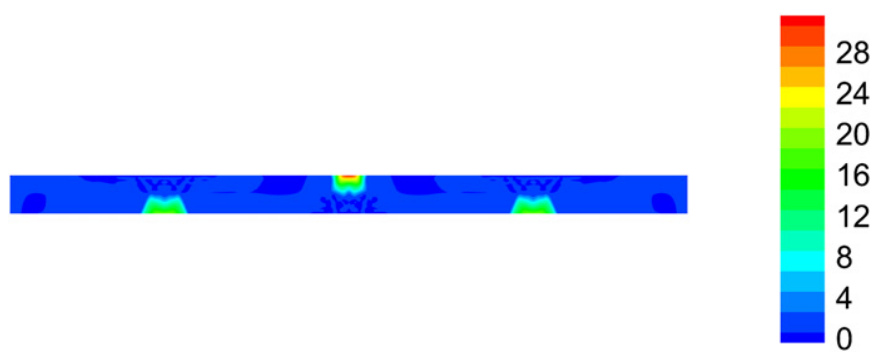

Fig. 12. Light bar: divergence of the velocity vector after the collision for $U^{-}=12.5 \mathrm{~m} / \mathrm{s}$. The fractures are represented by zone with high values of $\operatorname{div} \vec{U}^{+}$.

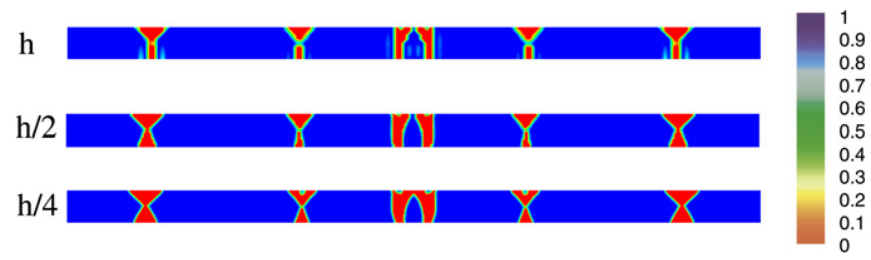

Fig. 13. Heavy bar: damage field for three meshes after the collision for $U^{-}=5 \mathrm{~m} / \mathrm{s}$.

opening mode (i.e., $\operatorname{div} \vec{U}^{+}>0$ ) or in compression regime (i.e., $\operatorname{div} \vec{U}^{+}=0$ ) while the fragmented regions are diffused damaged zones mainly due to very high strain rate.

The discrete solutions, obtained via finite element method, involve the resolution of a non-linear nonconvex minimization problem. The solution is obtained by an iterative calculation with a sequence of decreasing parameter $q \rightarrow 1$ starting from a quadratic convex term $(q=2)$. Specially, the calculation can be stopped at any iterative step and restarted from the previous solution even with an updated parameter $q$. This technique demonstrates to considerably speed up numerical convergence. Kinematical constraints are introduced by duality via the well known technique of the Lagrange multipliers. An ad-hoc iterative Uzawa algorithm has been developed for the resolution of the resulting saddle point problem due to the constraint inequalities.

Moreover, we restrict our attention to the case of 2-dimensional problems in plane strain condition of a single deformable body coming in contact with a rigid, fixed obstacle. The discretization is composed by quadrilateral finite element characterized by linear displacement and constant pressure inside the element, i.e., the so called Q1 $-P 0$ finite element (Braides and Fortin, 1991; Arnold et al., 2002) We also report a mesh sensitivity analysis in order to prove mesh objectivity of the predictive theory and of the numerical strategy adopted.

\subsection{Falling bar}

We consider the case of a slender rectangular bar colliding three rigid supports. Prior the collision, the bar is assumed to be undamaged $\left(\beta^{-}=1\right)$ and falls with a rigid body vertical descending velocity (see Fig. 1). The predictive theory gives the velocity and the damage after collision, $\vec{U}^{+}(\vec{x})$ and $\beta^{+}(\vec{x})$, depending on falling velocity $\vec{U}^{-}$. Two representative cases have been analyzed: all the parameters are fixed except the density of the material thus a heavy material and a light material, having the same resistance, have been considered (a ratio between the material densities equal to 10 has been adopted).

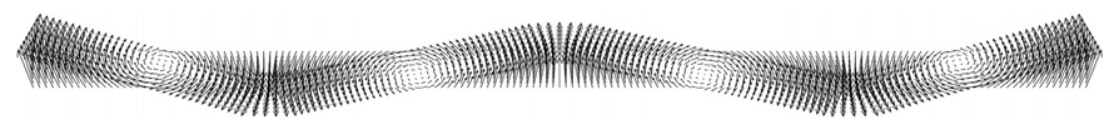

Fig. 11. Light bar: velocity $\vec{U}^{+}$after the collision for $U^{-}=12.5 \mathrm{~m} / \mathrm{s}$. The bar is broken into four pieces. 

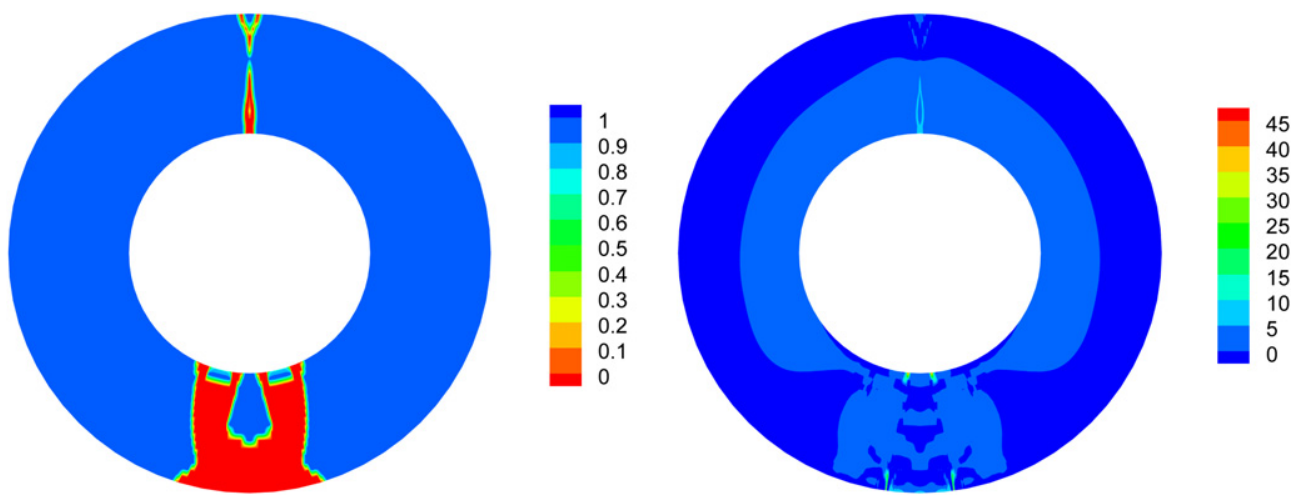

Fig. 14. Damage field $\beta^{+}$and divergence of the velocity field $\operatorname{div} \vec{U}^{+}$after collision. The adopted parameters have the following value: $\mu=8 . e 1 \mathrm{MPa}, \lambda=1 . e 1 \mathrm{MPa}, \lambda_{s}=2 . e 3 \mathrm{MPa} / \mathrm{mm}$, $3 . e-6 \mathrm{~N} / \mathrm{mm}^{3}, p=0.5, q=1.01, c=1 . e 3 \mathrm{MPa} \mathrm{s}, k=0.1 \mathrm{MPa} \mathrm{mm} \mathrm{mm}^{2}, w=1 . e 1 \mathrm{MPa}$.
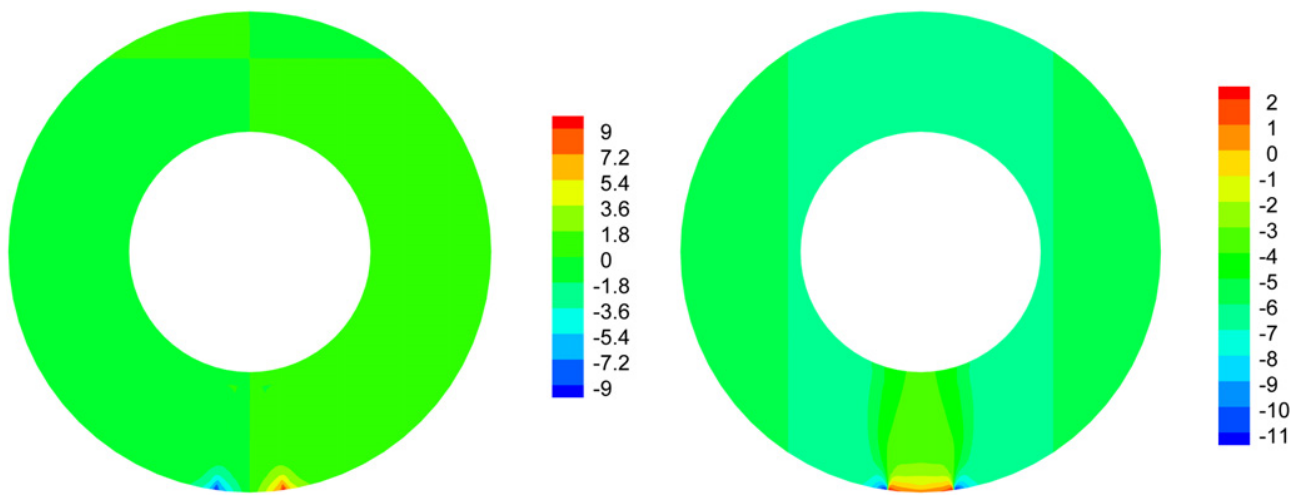

Fig. 15. Horizontal and vertical components $U_{x}^{+}$and $U_{y}^{+}$of the velocity field after collision. A zoom of the horizontal component at the top of the disk is shown in Fig. 16 .

Fig. 2 shows the damage field $\beta$ for different incoming velocities for the case of the heavy material. Fig. 2 clearly outcomes that the failure process can be classified with three states depending on the amount of imparted energy proportional to $\left(\vec{U}^{-}\right)^{2}$ : not fractured, fractured and fragmented states with rather sharp transitions in between. For a value of $U^{-}$smaller than $1 \mathrm{~m} / \mathrm{s}$, no fracture appears in the solid. For $1.5625 \leq U^{-} \leq 3.125 \mathrm{~m} / \mathrm{s}$ well defined fractures appear in the solids. It may be noted that the fractured portions of solid in compression are larger than those in traction. Intermediate incoming velocity value $6.25 \leq U^{-} \leq 12.5 \mathrm{~m} / \mathrm{s}$ outlines the

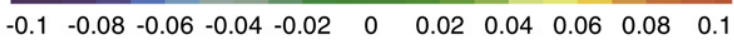

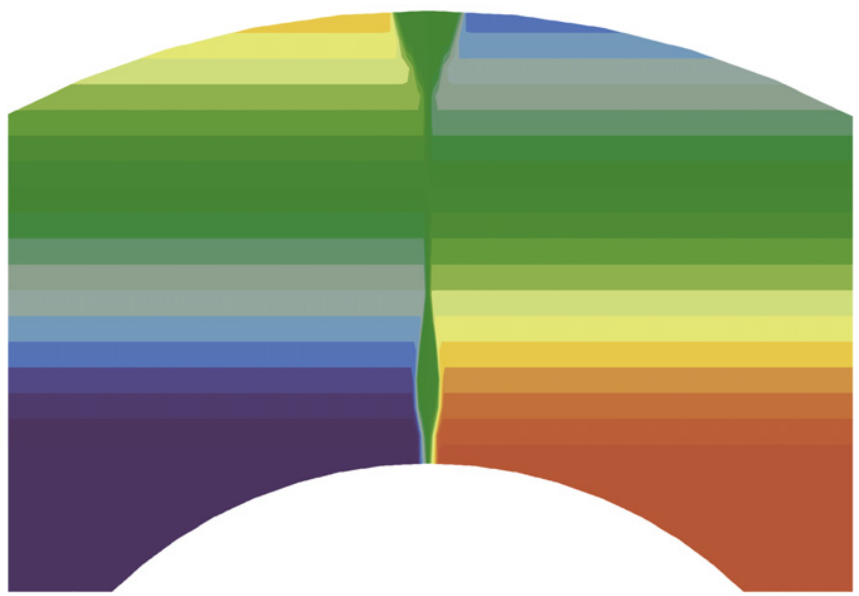

Fig. 16. Zoom of the horizontal component at the top of the disk. coexistence of the fractured and fragmented states. In fact, if fragmented regions appear after collision above the rigid supports, well defined fractures are still present inside the solid. Differently, high values of the impact velocity do not allow for the creation of well defined fractures and only fragmentation due to the large percussion transmitted between the solid and the rigid obstacles is present. It is important to note that the fragmented parts of the solids do not change significantly passing from $U^{-}=25 \mathrm{~m} / \mathrm{s}$ to $U^{-}=50 \mathrm{~m} / \mathrm{s}$ even if the kinetic energy becomes four times higher.

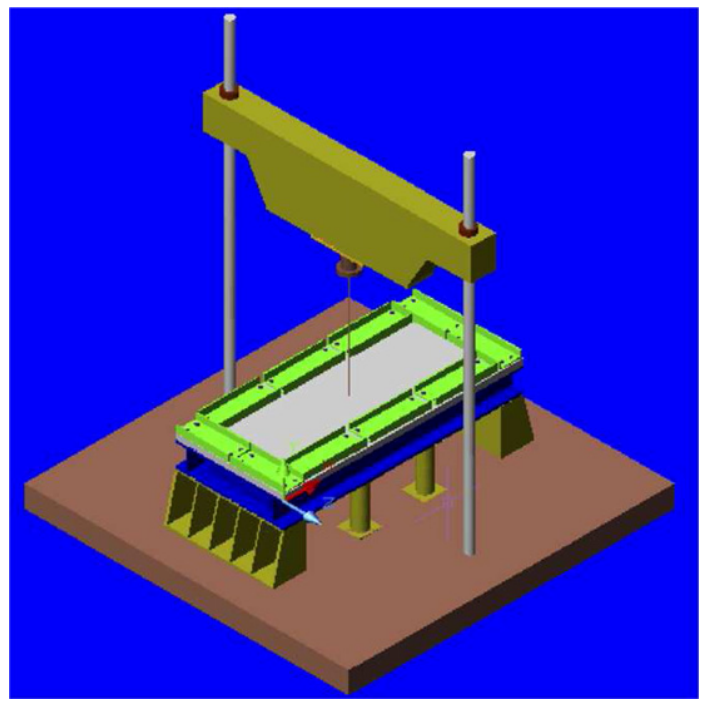

Fig. 17. Precision impact testing system proposed in Zineddin and Krauthammer (2007). 


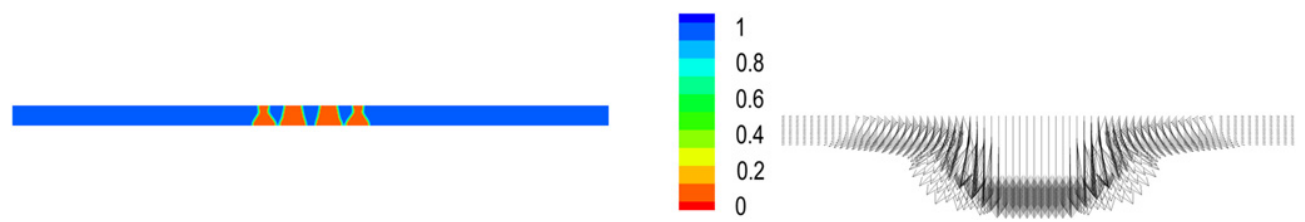

Fig. 18. Damage $\beta^{+}$and velocity field $\vec{U}^{+}$after collision. The adopted parameters have the following value: $\mu=5 . e 2 \mathrm{MPa}, \lambda=1 . e 1 \mathrm{MPa}, \rho=2.5 e-5 \mathrm{~N} / \mathrm{mm}{ }^{3}, p=0.5, q=1.01$, $c=3 . e 3 \mathrm{MPa} \mathrm{s}, k=0.1 \mathrm{MPa} \mathrm{mm}^{2}, w=1 . e 1 \mathrm{MPa}$.

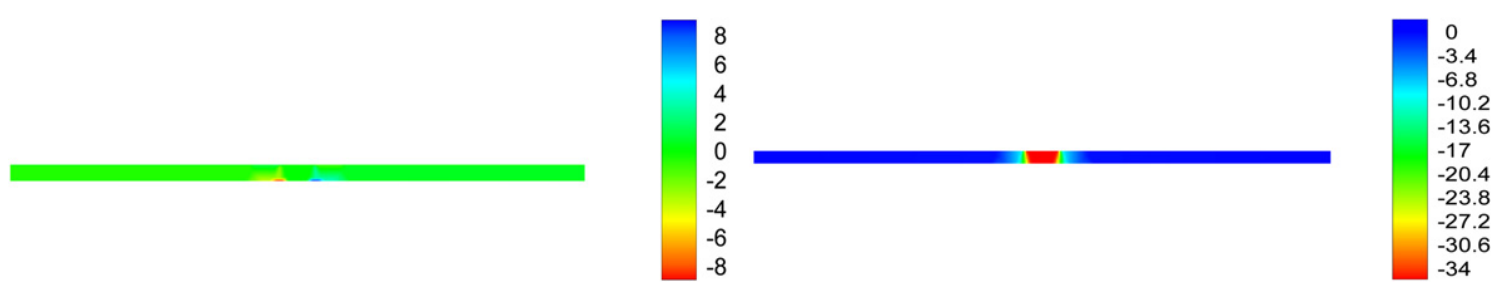

Fig. 19. Horizontal and vertical components $U_{x}^{+}$and $U_{y}^{+}$of the velocity field after collision.

In Fig. 3 the velocity field $\vec{U}^{+}$is reported for the case $U^{-}=1.5625 \mathrm{~m} / \mathrm{s}$. The bar is broken into five pieces after the collision. A central block stops above the central obstacle while instantaneous rigid rotations in the other block are induced by fracture creations.

This fact is shown by the representation of the horizontal velocity along two straight horizontal lines located at different positions reported in Fig. 4. Strong discontinuities in the velocity behaviour are localized in the fractured regions while the remaining pieces of the solid undergo a nearly rigid body motions. Moreover, the fractures in opening mode are outlined by the representation of the divergence of the velocity field reported in Fig. 5.

Similar considerations may be done for the case of $U^{-}=3.125 \mathrm{~m} / \mathrm{s}$ reported in Figs. 6 and 7 except by the fact that the bar breaks into seven pieces.

Fig. 8 represented the damage field $\beta$ for different incoming velocities for the case of the light material. In Figs. 9-12 the velocity fields and $\operatorname{div} \vec{U}^{+}$for two different values of falling velocity $U^{-}=6.25,12.5 \mathrm{~m} / \mathrm{s}$ are reported. The main differences with respect to the heavy material case can be summarized as follow:

- because of minor weight of the body, rebounds occur on the obstacles. This a general property of collisions both theoretical and experimental (Frémond, 2007);

- the central crack is completely different in this case: it is induced by rebound of the central portion;

- for high velocity values the light body breaks in several parts while the heavy material is cut into two elements.

\subsubsection{Mesh objectivity}

In this example we show the lack of mesh sensitivity of the predictive theory. The case of the heavy bar falling at a velocity of $5 \mathrm{~m} / \mathrm{s}$ is solved with different meshes to investigate whether or not the solutions converge. The computations are carried out for three structured meshes with different element sizes $\bar{h}, \bar{h} / 2$ and $\bar{h} / 4$ starting from the initial value $\bar{h}=20 \mathrm{~mm}$. The damage field evaluated for the three meshes is reported in Fig. 13 showing no mesh sensitivity with regard to the global solution of the problem and convergence to a solution is clearly represented. The velocity field after collision presents negligible differences.

\subsection{Falling annular disk}

The proposed model has been applied to the ideal case of an annular disk falling with a rigid vertical velocity and colliding with a fixed rigid floor. The annular disk has been decomposed with
20,000 finite elements. Fig. 14 shows the damage field, the divergence of the velocity field $\vec{U}^{+}$after the collision and the horizontal and the vertical components of the velocity field. We only underline the fact that two failure zones appear in the ring after collision. A first major fragmented zone is above the contact surface and involves the entire thickness of the annulus (see Fig. 15) while in the opposite part a well defined fracture is produced along the vertical direction due to a small but conspicuous discontinuity of the horizontal velocity field as outlined in Fig. 16.

\subsection{Impact simulation}

As a third example we reproduced qualitatively the impact tests reported in Zineddin and Krauthammer (2007). They illustrated different impact tests on reinforced concrete slabs with the machine reported in Fig. 17. The aim of this example is to show the capability of the proposed model to simulate actual experiments even if we do not compare numerical results with experimental evidences. First of all, we consider a simplified 2D example composed of 18,000 finite elements. Left and right extremities are clamped while the colliding mass is replaced by an external percussion applied on the upper surface of the slab. Moreover, we do not take into account the metallic reinforcements. Fig. 18 shows the damage field, the divergence of the velocity field $\vec{U}^{+}$after the collision and the horizontal and vertical components of the velocity field. Different failures are outlined from the numerical result in accordance with actual experiment. In fact, minor and major cracks are evidenced by the map of the velocity field reported in Fig. 19.

\section{Conclusions}

In this predictive theory for collisions and fractures only macroscopic quantities are involved. The equations of motion are derived from the principle of virtual work where new interior forces are introduce to describe the very large stresses and the very large contact forces resulting from the cinematic incompatibilities. The theory of collisions and fracture of solids outlined in this paper is consistent from the mechanical point of view and has good mathematical formulations to which adapted numerical methods may be applied. In the adopted collision theory the position of solid does not change but the velocity changes instantaneously. The acceleration at collision time is the velocity discontinuity. The constitutive laws which appear in this nonsmooth situation sum up the very sophisticated and rapid phenomena which occur in collision. 
Some numerical simulations have been performed via the classical finite element method, concerning a solid colliding against a rigid fixed obstacle or a clamped solid subjected to high velocity impact. The numerical results outlined multiple failure modes. Let us also stress that few parameters are involved, less than 9, to predict at the engineering level complex solid fracturation.

\section{References}

Arnold, D.N., Boffi, D., Falk, R.S., 2002. Approximation by quadrilateral finite elements. Math. Comp. 71, 909-922.

Ambrosio, L., Fusco, N., Pallara, D., 2000. Special Functions of Bounded Variations and Free Discontinuity Problems. Oxford University Press, Oxford.

Ambrosio, L., Coscia, A., Maso, G.D., 1997. Fine properties of functions with bounded deformation. Arch. Rational Mech. Anal. 139, 201-238.

Attouch, H., Buttazzo, G., Michaille, G., 2004. Variational Analysis in Sobolev and BV Spaces. Application to PDE and Optimization. In: MPS/SIAM Series in Optimization.

Bonetti, E., Frémond, M., 2004a. Collisions and fractures: a $1-D$ example. How to tear off a chandelier from the ceiling. J. Elasticity 74 (1), 47-66.

Bonetti, E., Frémond, M., 2004b. Collisions and fractures: a model in SBD. Rend. Mat. Acc. Lincei 15 (9), 47-57.

Braides, A., 1998. Approximation of Free-discontinuity Problems. Springer-Verlag, Berlin.

Braides, F., Fortin, M., 1991. Mixed and Hybrid Finite Element Methods. SpringerVerlag, New York.

Ciarlet, P.G., Lions, J.L., 1990. Handbook of Numerical Analysis. In: Handbook of Numerical Analysis, vol. I North-Holland, Amsterdam.
Fourney, W.L., 1993. Mechanisms of Rock Fragmentations by Blasting, vol. 4. Pergamon, Oxford, pp. 39-69.

Frémond, M., 1987. Adhérence des solides. J. de Mécanique théorique appliquée 6, 383-407.

Frémond, M., 2002. Non-smooth Thermomechanics. Springer Verlag, Heidelberg.

Frémond, M., 2007. Collisions. Edizioni del Dipartimento di Ingegneria Civile dell'Università di Roma Tor Vergata, ISBN 978-88-6296-000-7.

Frémond, M., Gormaz, R., Martín, J.A., 2003. Collision of a solid with an incompressible fluid. Theor. Comput. Fluid Dynam. 16, 405-420.

Frémond, M., Nedjar, B., 1996. Damage, gradient of damage and principle of virtual power. Int. J. Solid Struct. 8, 1083-1103.

Friaa, A. 1979. La loi de Norton-Hoff généralisée en plasticité et en viscoplasticité. PhD thesis, University Pierre and Marie Curie, Paris, France.

Halphen, B., Son, N.Q., 1975. Sur les matériaux standards généralisés. J. de Mécanique 14.

Moreau, J.J., 1966b. Principes extrêmaux pour le problème de la naissance de la cavitation. J. de Mécanique 5, 439-470.

Moreau, J.J., 2003. Fonctionelles Convexes. Edizioni del Dipartimento di Ingegneria Civile dell'Università di Roma Tor Vergata, ISBN 978-88-6296-001-4.

Moreau, J.J., 1970. Sur les lois du frottement, de viscosité et de plasticité. C. R. Acad. Sci., Paris 271, 608-611.

Romero, H. 2003. Fragmentation du béton sous explosion. PhD thesis, Université Montpellier II, Montpellier, France.

Snyman, J.A., 2005. Practical Mathematical Optimization: An Introduction to Basic Optimization Theory and Classical and New Gradient-based Algorithms. Springer Verlag.

Zineddin, M., Krauthammer, T., 2007. Dynamic response and behavior of reinforced concrete slabs under impact loading. Int. J. Impact Eng. 34, 1517-1534.

Zulehner, W., 2002. Analysis of iterative methods for saddle point problems: a unified approach. Math. Comp. 71, 479-505. 